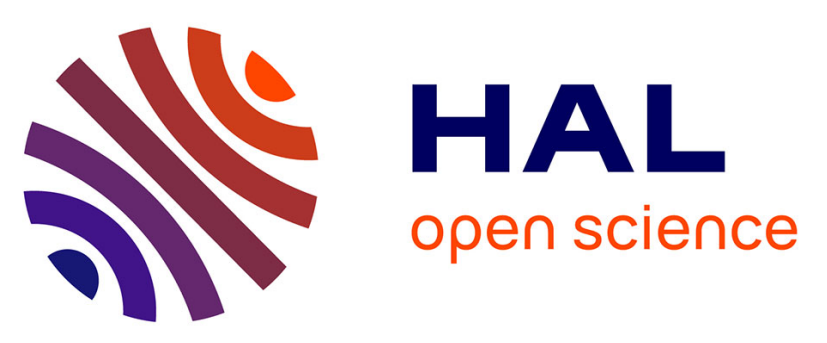

\title{
Simulation of the flow past a circular cylinder in the supercritical regime by blending RANS and variational-multiscale LES models
}

Carine Moussaed, Maria Vittoria, Stephen Wornom, Bruno Koobus, Alain Dervieux

\section{To cite this version:}

Carine Moussaed, Maria Vittoria, Stephen Wornom, Bruno Koobus, Alain Dervieux. Simulation of the flow past a circular cylinder in the supercritical regime by blending RANS and variational-multiscale LES models. Journal of Fluids and Structures, 2014, 47, pp.114 - 123. 10.1016/j.jfluidstructs.2013.11.006 . hal-01083801

\section{HAL Id: hal-01083801 https://hal.inria.fr/hal-01083801}

Submitted on 18 Nov 2014

HAL is a multi-disciplinary open access archive for the deposit and dissemination of scientific research documents, whether they are published or not. The documents may come from teaching and research institutions in France or abroad, or from public or private research centers.
L'archive ouverte pluridisciplinaire HAL, est destinée au dépôt et à la diffusion de documents scientifiques de niveau recherche, publiés ou non, émanant des établissements d'enseignement et de recherche français ou étrangers, des laboratoires publics ou privés. 


\title{
Simulation of the flow past a circular cylinder in the supercritical regime by blending RANS and variational-multiscale LES models
}

Carine Moussaed ${ }^{\mathrm{a}}$, Maria Vittoria Salvetti ${ }^{\mathrm{b}}$, Stephen Wornom ${ }^{\mathrm{c}}$, Bruno Koobus $^{\mathrm{a}}$, Alain Dervieux ${ }^{\mathrm{d}}$

a I3M, Université Montpellier 2, Case Courrier 051, Place Eugène Bataillon, 34095

Montpellier, France.

${ }^{b}$ Dipartimento di Ingegneria Aerospaziale, Università di Pisa, Via G. Caruso 8, 56122 Pisa, Italy.

${ }^{c}$ LEMMA, 2000 Route des Lucioles, 06902 Sophia-Antipolis, France.

${ }^{d}$ INRIA, 2004 Route des lucioles, BP 93, 06902 Sophia Antipolis, France.

\begin{abstract}
A strategy which blends a Variational Multiscale Large Eddy Simulation model (VMS-LES) and a RANS model in a hybrid approach is investigated. A smooth blending function, which is based on the value of a blending parameter, is used for switching from VMS-LES to RANS. Different definitions of the blending parameter are investigated. The capabilities of the novel hybrid approach are appraised in the simulation of the flow around a circular cylinder at a Reynolds number $1.4 \cdot 10^{5}$, based on the freestream velocity and on the cylinder diameter, in presence of turbulent boundary-layer due to turbulent inflow conditions. A second study at Reynolds numbers from $6.7 \cdot 10^{5}$ to $1.25 \cdot 10^{6}$ is also presented. The effect of using the VMS-LES approach in the hybrid model is evaluated. Results are compared to those of other RANS, LES and hybrid simulations in the literature and with experimental data
\end{abstract}

Keywords:

Hybrid RANS/VMS-LES approach, bluff-body flows, unstructured grids, circular cylinder, supercritical regime 


\section{Introduction}

The widely used RANS models usually have difficulties in providing accurate predictions for flows with massive separation, as for instance the flow around bluff bodies. An alternative approach is Large-Eddy Simulation (LES), which, for massively separated flows, is generally more accurate, but also computationally more expensive, than RANS. Indeed the LES grid needs to be sufficiently fine to resolve a significant part of the turbulent scales, and this becomes particularly critical in the near-wall regions. Moreover, the cost of LES increases with increasing Reynolds number. In this context, hybrid strategies have been proposed in the literature, which combine RANS and LES approaches together (see [28],[9] for a review).

A major difficulty in combining a classical RANS model with a LES one is due to the fact that they deal with different unknowns. The RANS equations govern an ensemble-averaged flow field. The LES equations describe the evolution of a spatially filtered flow field. RANS solutions are often steady or contain only the unsteadiness of the largest scales, if large-scale unsteadiness is present in the flow (which is true for bluff-body flows). On the other hand, LES needs a significant level of fluctuations in order to model the flow in a sufficiently accurate manner. Attempts to interface RANS and LES are faced to this problem (see e.g. [33, 28]). Recently, temporal filtering has been proposed as a possible way to make these two models more compatible [7]. However, even in their standard version, hybrid models have acquired a remarkable standing in the simulation of detached turbulent flows, since they often produce much better predictions than pure RANS, with a much more affordable computational effort than for pure LES, cf. [36]. In the present work, we do address this issue.

A central issue in hybrid modeling is also the selection of RANS and LES regions. It is commonly accepted that, for affordable levels of discretization, RANS is advisable in a turbulent boundary layer and LES for detached eddies in the wake of a bluff body. We are interested in appraising hybrid approaches which could eventually be applied in an engineering context to the simulation of massively separated flows in complex geometries. In this perspective, hybridization strategies in which the LES and RANS zones may be selected during the simulation following a given criterion, and not a-priori fixed, may be attractive. Clearly, for such hybrid methods, it must be aposteriori checked that the automatic selection of the RANS and LES zones is indeed effective. Moreover, depending on the local mesh resolution and 
on the geometry complexity, it remains important to investigate methods for selecting the less inexact model in tricky regions of the flow, as for instance the detaching shear layers in bluff-body flows.

This is in relation with a third issue, which concerns the choice of the main ingredients in the hybrid modeling, viz. the RANS model, and the LES one. Again in the perspective of the use of a hybridization strategy in an engineering context, a desirable feature would be versatility, i.e. the possibility of straightforwardly introducing different RANS and LES models. In particular it can also be of interest to introduce LES formulations different from the classical one. For example, the Variational Multiscale (VMS) LES showed in $[39,25]$ good predictive qualities for subcritical flows around bluff bodies also with rather coarse meshes.

In the present work, a hybrid model blending RANS and Variational Multiscale (VMS) LES [18] is investigated. In this model, VMS-LES and RANS approaches are combined in such a manner that complexity in use and in parameter tuning is minimized. The closure terms provided by a RANS and a SGS eddy-viscosity model are blended together through the introduction of a blending function, $\theta$, obtaining the RANS approach when $\theta=1$ and recovering the LES approach for $\theta$ vanishing. Thus, the adopted model can be thought to fall in the class of the blending methods $[16,8,3]$, following the definition in [28]. We investigate different blending parameters aimed at identifying whether the grid used is adequate for LES, i.e. if it is adequate to resolve additional fluctuations with respect to RANS. In particular, three different definitions are examined in this paper, based on the ratios between (i) two eddy viscosities, (ii) two characteristic length scales and (iii) two characteristic time scales given by the RANS and the LES models, respectively. One of the aim of the present work is indeed to check whether this criterion, more focused on grid quality than directly to the wall distance, actually gives the desired situation of the RANS model working near solid walls. Note that the criterion (i) is used also in [3], but only as an additional constraint to the main criterion based on the distance from the wall, and is actually the parameter of the "Limited Numerical Scale" (LNS) approach [2], in which, however, a different hybridization strategy is used.

An additional attractive feature of the investigated blending strategy is that it permits a natural integration of the VMS concept, which allows the eddy-viscosity introduced by the LES closure to be restricted to the smallest resolved scales. This is aimed at reducing the excessive damping introduced by non-dynamic eddy-viscosity models also on large scales. 
Our motivation in combining RANS with the VMS formulation is to be able to perform turbulent flow simulations on coarser meshes than with RANS/LES hybrid model with a comparable accuracy. This is a paramount advantage if complex geometries of several obstacles are to be analysed, such as tube arrays (see e.g. [21]) or offshore platforms, see [31]. Lastly, in our opinion, the VMS-LES approach well fits in a multidimensional turbulence modeling concept as in [28].

The above described hybrid method is herein applied to the simulation of the flow around a circular cylinder of infinite span in different conditions. First, the case proposed in [36] is considered, which is characterized by a Reynolds number - based on the freestream velocity and the cylinder diameter - equal to $1.410^{5}$ and by highly turbulent inflow conditions. These inflow conditions, together with the use of a RANS model in the boundary layer, allow a turbulent boundary-layer separation to be obtained even if the Reynolds number is not in the supercritical regime. This test case has been chosen in order to make a direct comparison with the results of DES simulations [20,36], DES being the most popular of hybrid approaches.

In a second series of computations, the flow around a cylinder has been carried out at Reynolds numbers close to $10^{6}$, in order to investigate the behavior of the considered hybrid methods in the actual supercritical regime. In the litterature, RANS or VLES methods are used for this case. We pay a particular attention to the comparison of these two options with our hybrid approach.

\section{Methodology}

The governing equations are discretized in space using a mixed finitevolume/finite-element method applied to unstructured tetrahedrizations. This scheme is a variational one relying on a finite-volume formulation for the convective terms, with a basis and test function $\chi_{l}$, associated with the finitevolume cell centered on vertex $l$. A finite-element formulation is used for the diffusive term, with a basis and test function $\phi_{l}$ continuous, linear by element, equal to 1 at vertex $l$ and vanishing at other vertexes. The Roe scheme [26] represents the basic upwind component for the numerical evaluation of the convective fluxes. A Turkel-type preconditioning term is introduced to avoid accuracy problems at low Mach numbers [12]. To obtain second-order accuracy in space, the Monotone Upwind Scheme for Conservation Laws reconstruction method (MUSCL) is used, in which the Roe flux is expressed as a 
function of reconstructed values of $W$ at each side of the interface between two cells. The introduced numerical dissipation is made of sixth-order space derivatives [4] and, thus, concentrates on a narrow-band of the highest resolved frequencies. Finally, an implicit linearized time-marching algorithm is used, based on a backward-difference scheme for the discretization of the time derivative. The numerical method is second-order accurate in space and time. More details can be found in [4].

The hybridization strategy is based on the NLDE idea, in which the flow variables are decomposed as follows [19]:

$$
W=\underbrace{\leqslant W \geqslant}_{\text {RANS }}+\underbrace{W^{c}}_{\text {correction }}+W^{S G S}
$$

where $\langle W\rangle$ are the RANS flow variables, obtained by applying an averaging operator to the Navier-Stokes equations, $W^{c}$ are the remaining resolved fluctuations (i.e. $\langle W\rangle+W^{c}$ are the resolved flow variables for LES or VMS-LES in our case) and $W^{S G S}$ are the unresolved or SGS fluctuations.

As far as the closure of the RANS equations is concerned, we use the Low Reynolds $k-\varepsilon$ model proposed in [11], which was designed to improve the predictions of the standard $k-\varepsilon$ one for adverse pressure gradient flows, including separated flows. The semi-discretization of the mean flow equations can be written down as:

$$
\begin{aligned}
& \left(\frac{\partial\langle W\rangle}{\partial t}, \chi_{l}\right)+\left(\nabla \cdot F_{c}(\langle W\rangle), \chi_{l}\right)+\left(\nabla \cdot F_{v}(\langle W\rangle), \phi_{l}\right)= \\
& -\left(\tau^{R}(\langle W\rangle), \phi_{l}\right) \quad l=1, N .
\end{aligned}
$$

in which $(\cdot, \cdot)$ denotes the $L^{2}$ scalar product and $F_{c}$ and $F_{v}$ are the convective and viscous fluxes. In this model, the Reynolds stress tensor $\tau^{R}$ has the same form as in the standard $k-\varepsilon$ model but the turbulent eddy viscosity is defined by $\mu_{t}=C_{\mu} f_{\mu} \frac{k^{2}}{\varepsilon}$ where $C_{\mu}=0.09$ and the damping function $f_{\mu}$ is given by $f_{\mu}=\frac{1-e^{-0.01 R_{t}}}{1-e^{-\sqrt{R_{t}}}} \max \left[1, \frac{\sqrt{2}}{\sqrt{R_{t}}}\right]$ with $R_{t}=\frac{k^{2}}{\nu \varepsilon} ; k$ and $\varepsilon$ are determined by ad-hoc modeled transport equations. In order to master mesh requirements, a wall law is applied in the close vicinity of the wall. For accuracy purpose, we choose the Reichardt analytical law [14] which gives a smooth matching between linear, buffer and logarithmic regions. Because the $y^{+}$normalized distance is generally subject to large variations in complex 
geometries, we have found mandatory to combine together wall law and low Reynolds modeling which locally damps the fully-turbulent model in regions in which the wall law does not cover the buffer zone.

For the LES part, we consider the VMS approach, in which the resolved flow variables are decomposed as $\widetilde{W}=<W>+W^{c}=\bar{W}+W^{\prime}$, where $\bar{W}$ are the large resolved scales (LRS) and $W^{\prime}$ are the small resolved scales (SRS). We follow here the VMS approach proposed in [18] for the simulation of compressible turbulent flows through a finite volume/finite element discretization on unstructured tetrahedral grids. In order to obtain the VMS flow decomposition, basis and test functions can be expressed as: $\chi_{l}=\bar{\chi}_{l}+\chi_{l}^{\prime}$ and $\phi_{l}=\bar{\phi}_{l}+\phi_{l}^{\prime}$, in which the overbar denotes the basis functions spanning the finite dimensional spaces of the large resolved scales and the prime those spanning the SRS spaces. As in [18], the basis functions of the LRS space are defined through a projector operator in the LRS space, based on spatial average on macro cells, which are obtained by an agglomeration process. Eddy-viscosity models are used here for the SGS terms, and more precisely those proposed by Smagorinsky [32], Vreman [37] and the so-called WALE model [23]. The eddy-viscosity introduced by these models, within the VMS approach, is computed as a function of the SRS flow variables (small-small approach). Moreover, the key feature of the VMS approach is that the SGS model is added only to the smallest resolved scales. Finally, the SGS terms are discretized analogously to the viscous fluxes. Thus, the Galerkin projection of the LES closure term is: $-\left(\tau^{L}\left(W^{\prime}\right), \phi_{l}^{\prime}\right)$, and the VMS-LES equations can then be written as follows:

$$
\begin{aligned}
& \left(\frac{\partial \widetilde{W}}{\partial t}, \chi_{l}\right)+\left(\nabla \cdot F_{c}(\widetilde{W}), \chi_{l}\right)+\left(\nabla \cdot F_{v}(\widetilde{W}), \phi_{l}\right)= \\
& -\left(\tau^{L}\left(W^{\prime}\right), \phi_{l}^{\prime}\right) \quad l=1, N .
\end{aligned}
$$

The filter width $\Delta$ involved in the definition of the eddy-viscosity has been set equal to the cubic root of the volume of each tetrahedron. The SGS model constant has been set equal to 0.1 for the Smagorinsky model, to 0.5 for WALE and to 0.025 for the Vreman one.

An equation for the resolved fluctuations $W^{c}$ can then be derived by substracting Eq. (1) to Eq. (2): 


$$
\begin{aligned}
& \left(\frac{\partial W^{c}}{\partial t}, \chi_{l}\right)+\left(\nabla \cdot F_{c}(\widetilde{W}), \chi_{l}\right)-\left(\nabla \cdot F_{c}(<W>), \chi_{l}\right)+\left(\nabla \cdot F_{v}\left(W^{c}\right), \phi_{l}\right)= \\
& \left(\tau^{R}(\langle W\rangle), \phi_{l}\right)-\left(\tau^{L}\left(W^{\prime}\right), \phi_{l}^{\prime}\right) \quad l=1, N .
\end{aligned}
$$

The basic idea of the proposed hybrid model is to solve Eq. (1) in the whole domain and to correct the obtained averaged flow by adding the remaining resolved fluctuations computed through Eq. (3), wherever the grid resolution is adequate for VMS-LES. To identify the regions where the additional fluctuations need to be computed, a blending function $\theta$ is introduced, which smoothly varies between 0 and 1 . For $\theta=1$, no correction to $\langle W\rangle$ is added, and the RANS approach is recovered. Conversely, wherever $\theta<1$, additional resolved fluctuations are computed, and we want to recover the VMS-LES approach as $\theta \rightarrow 0$. Thus, the equation for the resolved fluctuations $W^{c}$ becomes:

$$
\begin{aligned}
& \left(\frac{\partial W^{c}}{\partial t}, \chi_{l}\right)+\left(\nabla \cdot F_{c}(\widetilde{W}), \chi_{l}\right)-\left(\nabla \cdot F_{c}(<W>), \chi_{l}\right)+\left(\nabla \cdot F_{v}\left(W^{c}\right), \phi_{l}\right)= \\
& (1-\theta)\left[\left(\tau^{R}(\langle W\rangle), \phi_{l}\right)-\left(\tau^{L}\left(W^{\prime}\right), \phi_{l}^{\prime}\right)\right] \quad l=1, N .
\end{aligned}
$$

Although it could seem rather arbitrary from a physical point of view, in Eq. (4) the damping of the RHS through multiplication by $(1-\theta)$ is aimed to obtain a progressive addition of fluctuations and, therefore, a progressive switch of the model from the RANS to the VMS-LES mode where the grid resolution is adequate.

Up to this point, following the NLDE approach, the hybrid method would imply the solution of two systems of equations (Eqs. (1) and (4)) to compute two different sets of variables, $\langle W\rangle$ and $W^{c}$. Clearly, although possible, this would lead to large computational costs. Therefore, we propose a further simplified version of the hybridization. To this aim, Eqs. (1) and (4) are recast together as follows:

$$
\begin{aligned}
& \left(\frac{\partial \widetilde{W}}{\partial t}, \chi_{l}\right)+\left(\nabla \cdot F_{c}(\widetilde{W}), \chi_{l}\right)+\left(\nabla \cdot F_{v}(\widetilde{W}), \phi_{l}\right)= \\
& -\theta\left(\tau^{R}(\langle W\rangle), \phi_{l}\right)-(1-\theta)\left(\tau^{L}\left(W^{\prime}\right), \phi_{l}^{\prime}\right) \quad l=1, N .
\end{aligned}
$$

In Eq. (5), $\langle W\rangle$ should be the RANS mean, which is not available from the solution of Eq. (5). In this study, we simply use $\tau^{R}(\widetilde{W})$, relying on the 
previously discussed tendency of RANS to naturally damp the fluctuations. More sophisticated options are possible but they are not considered in the present paper, again with the aim of obtaining a hybrid method suitable for complex engineering simulations.

As blending function $\theta$, we use $\theta=\tanh (\xi)$, where $\xi$ is the blending parameter, which should indicate whether the grid resolution is fine enough to resolve a significant part of the turbulence fluctuations, i.e. to obtain a LES-like simulation. The choice of the blending parameter is clearly a key point for the definition of the present hybrid model. In the present study, different options are proposed and investigated, namely: the ratio between the eddy viscosities given by the LES and the RANS closures, $\xi_{V R}=\mu_{s} / \mu_{t}$, the ratio between the LES filter width and a typical length in the RANS approach, $\xi_{L R}=\Delta / l_{R A N S}$ with $l_{R A N S}=k^{3 / 2} \epsilon^{-1}$, and, finally, the ratio between characteristic times of the LES and RANS approaches, $\xi_{T R}=t_{L E S} / t_{R A N S}$ with $t_{L E S}=\left(S_{i j} S_{i j}\right)^{-1 / 2}$ and $t_{R A N S}=k \epsilon^{-1}$. The blending parameter clearly depends on the grid resolution, since, as previously stated, the aim ideally is to obtain a progressive switch from URANS to LES where the grid resolution becomes fine enough to resolve a significant part of the local turbulence scales or fluctuations. The effects of grid resolution will be addressed in the following Section.

\section{Supercritical flow around a circular cylinder at $R e=140000$}

The proposed approach has been applied to the simulation of the flow around a circular cylinder at $R e=140000$ (based on the far-field velocity and the cylinder diameter, $D$ ). This is a case proposed in [36]. While at this Reynolds number the flow should be subcritical, the peculiarity of this test case is that the flow is forced to be supercritical, by using a RANS model in the boundary layer and increasing the turbulence intensity inflow condition. In our computation, the domain dimensions are $L_{i} / D=5, L_{o} / D=15$, $L_{y} / D=14$ where $L_{z} / D=2, L_{i}$ and $L_{o}$ are the distances of the cylinder center from the domain inflow and the outflow, and $L_{y}$ and $L_{z}$ the domain sizes in the lateral and spanwise directions. On the side surfaces free-slip is imposed and the flow is assumed to be periodic in the spanwise direction. The inflow Mach number is set to 0.1 so that the compressibility effects can be considered as negligible. The inflow value of eddy-viscosity is set to about 5 times the molecular viscosity. The Reichardt analytical wall function is used

on the cylinder surface. We refer e.g. to [6] for details on the computation of the turbulence variables from a wall law. 
Two different grids are considered; the first one (GR1) has $4.6 \times 10^{5}$ nodes, while the second one has (GR2) $1.4 \times 10^{6}$ nodes. Both grids are composed of a structured part in a circular crown around the cylinder surface and a unstructured part in the rest of the domain. Inside the structured zone, the nodes are uniformly distributed in the azimuthal and spanwise directions, with 180 and 40 nodes for GR1 and 360 and 80 for GR2. On the other hand, the radial distribution of nodes inside the structured zone obey a geometric progression and the resolution is the same for both grids, with a distance between the the cylinder boundary and the nearest layer of $0.004 D$. From a rough estimation carried out by using an average shear velocity, this corresponds to $y^{+} \approx 28$. However, as previously mentioned, the $y^{+}$is actually significantly varying since a strong adverse pressure gradient is present. Note that, to limit the total number of nodes, for GR2 the radial thickness, $r t$, of the structured crown is half of that for GR1: $r t / D=0.1$ for GR1 and $r t / D=0.05$ for GR2.

The main bulk flow parameters obtained in the hybrid simulations are summarized in Table 1, together with the results of DES simulations in the literature and some experimental data. The names of the hybrid simulations indicate: first the grid (GR1 or GR2), then the blending parameter (VR and LR for viscosity ratio and length ratio) and finally the SGS model (S, V and $\mathrm{W}$ for Smagorinsky, Vreman and Wale). The results obtained in a 3D URANS simulation carried out with the Low-Reynolds $k-\varepsilon$ model [11] on the GR1 grid are also reported.

As a general consideration, the agreement with the DES simulations, with which our results can be directly compared, is good for all the simulations carried out with the present hybrid approach. As for the comparison with the experiments, since our simulations are characterized by turbulent separation, it makes sense to compare the results with experiments at higher Reynolds number, as also done in [36], in which the transition to turbulence of the boundary layer also occurs upstream separation. The agreement with these high Re experiments is indeed satisfactory, as shown in Table 1 and in Figure 1, showing the distribution of the mean pressure coefficient over the cylinder surface. Conversely, the results of the URANS simulation show significant discrepancies with respect to the DES and the experimental data and, namely, a noticeably higher absolute value of the $C_{p}$ in the wake, which results in an overestimated mean drag coefficient. Moreover, the mean recirculation bubble is significantly shorter than in the reference experiments and in the hybrid simulations, and this is connected with an overestimation 
Table 1: Main bulk flow quantities. $\overline{C_{d}}$ is the mean drag coefficient, $C_{l}^{\prime}$ is the r.m.s. of the lift coefficient, $S t$ the vortex shedding frequency made nondimensional by the cylinder diameter and the freestream velocity, $l_{r}$ is the length of the mean recirculation bubble and $C_{p b}$ the value of the mean base pressure coefficient. Blending parameter: VR and LR for viscosity ratio and length ratio. SGS model: S, V and W for Smagorinsky, Vreman and Wale.

\begin{tabular}{|c|c|c|c|c|c|c|}
\hline $\begin{array}{l}\text { Present } \\
\text { Simulations }\end{array}$ & $R e$ & $\overline{C_{d}}$ & $C_{l}^{\prime}$ & $-C_{p b}$ & St & $l_{r}$ \\
\hline GR1-URANS & $1.410^{5}$ & 0.756 & 0.433 & 0.85 & 0.275 & 0.67 \\
\hline GR1-LRV & $1.410^{5}$ & 0.65 & 0.077 & 0.70 & 0.28 & 1.14 \\
\hline GR1-LRW & $1.410^{5}$ & 0.66 & 0.094 & 0.72 & 0.28 & 1.24 \\
\hline GR1-VRS & $1.410^{5}$ & 0.62 & 0.083 & 0.72 & 0.30 & 1.20 \\
\hline GR1-LRS & $1.410^{5}$ & 0.62 & 0.083 & 0.68 & 0.30 & 1.19 \\
\hline GR2-LRS & $1.410^{5}$ & 0.54 & 0.065 & 0.63 & 0.33 & 1.13 \\
\hline \multicolumn{7}{|l|}{ Simulations } \\
\hline DES [36] & $1.410^{5}$ & $0.57-0.65$ & $0.06-0.1$ & $0.65-0.7$ & $0.28-0.31$ & $1.1-1.4$ \\
\hline DES [20] & $1.410^{5}$ & $0.6-0.81$ & - & $0.85-0.91$ & $0.29-0.3$ & $0.6-0.81$ \\
\hline \multicolumn{7}{|l|}{ Measurements } \\
\hline Exp. [15] & $3.810^{6}$ & 0.58 & - & 0.58 & 0.25 & - \\
\hline Exp. [1] & $510^{6}$ & 0.7 & - & - & - & - \\
\hline Exp. [27] & $8.410^{6}$ & 0.7 & - & 0.8 & 0.27 & - \\
\hline Exp. [29] & $810^{6}$ & 0.52 & 0.06 & - & 0.28 & - \\
\hline
\end{tabular}


of the amplitude of lift oscillations (see the values of $C_{l}^{\prime}$ in Table 1). An example of the time behavior of the lift coefficient for a hybrid simulation (GR1-LRS) is given in Figure 2a and can be compared with the one obtained in the URANS simulation shown in Figure 2b. Besides the larger amplitude of the $C_{l}$ oscillations, it is evident that in the URANS simulation the modulations of the amplitude of these oscillations are significantly less important than in the hybrid simulations. The lift behavior obtained in the URANS simulation is close to those typically obtained in 2D simulations (e.g. [22]); indeed in the present URANS simulation the 3D phenomena in the flow have been observed to be almost totally damped because of a too large introduced turbulent viscosity. This is confirmed also by the instantaneous field values of the spanwise velocity and of the vorticity components $\omega_{x}$ and $\omega_{y}$, which are much lower in the RANS simulation than in the hybrid ones (not shown here for sake of brevity).

As for the behavior of the hybridization strategy in the field, for all the definitions of the blending parameter, the model actually works in RANS mode in the boundary layer and in the shear-layers detaching from the cylinder, while in the wake a full LES correction is recovered. This is shown, for instance, in Figure 3, in which the instantaneous isocontours of spanwise vorticity obtained in the simulation GR1-LRS are reported, to which the isolines of the blending function $\theta=0.1$ and $\theta=0.9$ are superimposed. This a-posteriori confirms that the introduced blending function is actually able to give, at least qualitatively, the desired behavior in hybrid simulations of bluff-body flows. From Figure 4, it appears that a smooth passage from a RANS modeling in the boundary layer and in the detaching shear-layers to a LES one in the wake is obtained, consistently with one of the aims of the investigated hybridization strategy.

Let us analyze now the sensitivity of the results obtained in the hybrid simulations to different parameters and, first, the sensitivity to the definition of the blending parameter, by comparing the results of the simulations GR1VRS and GR1-LRS. From Table 1, it appears that the results are practically insensitive to the definition of the blending parameter. This is also confirmed, for instance, by the distribution over the cylinder of the mean pressure coefficient, $C_{p}$, reported in Fig.1a. As for the behavior of hybridization strategy, it is also similar for the two definitions of the blending parameter. Analogous results were also found with the definition based on the ratio of characteristic times and they are not shown here for sake of brevity. Moreover, the sensitivity to the VMS-LES closure model is very low (compare GR1-LRS, 
GR1-LRV and GR1-LRW in Table 1 and Figure 1b).

As for the effect of grid refinement, from Table 1 it can be seen that it leads to an increase of the Strouhal number of about $10 \%$ with respect to the simulations on the coarser grid. The obtained value is significantly larger than the experimental ones reported in Table 1. These experimental data are however only some examples of those available in the supercritical regime, and the Strouhal values obtained in our simulations are inside the experimental range in this regime (see e.g. [40]). Grid refinement also leads to a decrease of $\bar{C}_{d}$ (compare GR1-LRS and GR2-LRS). This is due to a decrease of the absolute value of the pressure coefficient in the separated wake (see Table 1 and Fig ure 1b). However, note that, as it is quite a usual situation for unstructured grids, the refinement carried out to obtain grid GR2 has significantly changed the local quality of the grid (in terms of homogeneity and regularity of the elements) and this may enhance the sensitivity of the results. As for the behavior of the hybridization strategy with grid refinement, it is qualitatively correct. Indeed, the extension of the zone in the detaching shear-layers in which the model works in RANS mode decreases with grid refinement, as shown for instance in Figure 4 reporting a zoom near the cylinder of the instantaneous isocontours of the blending function $\theta$, obtained in the simulations GR1-LRS and GR2-LRS. This is consistent with the spirit of the blending method; indeed, for a finer grid resolution a larger part of the grid is detected as adequate for LES and thus the RANS zone is reduced.

\section{Supercritical flow around a circular cylinder at $R e=1 M$}

Only a few numerical investigations have been performed for Reynolds numbers higher than $5 \cdot 10^{5}$. This interval is inside the supercritical regime which appears at Reynolds number higher than $2 \cdot 10^{5}$ and for which the separation becomes turbulent, see [34]. It is generally accepted that periodic vortex shedding is very weak (if any) in the lowest parts of the interval $\left[5 \cdot 10^{5}, 1.5 \cdot 10^{6}\right]$. With increasing Reynolds numbers, the final separation point moves forward, which corresponds to a slight increase of the drag.

We carried out simulations at three different Reynolds number, viz. 6.7 . $10^{5}, 10^{6}$ and $1.25 \cdot 10^{6}$.

For these simulations, the computational domain is a cylinder of diameter 20 times larger than the obstacle, and with a span of $\pi$ times the obstacle diameter. The boundary conditions are the same as for the case at $R e=$ 
$1.4 \cdot 10^{5}$, except for the inflow, at which the turbulent viscosity is set to 30 times the molecular viscosity. The wall law approach is used with a distance to the wall $\delta$ set to .002 , which corresponds for the most part to $y^{+} \simeq 100$. Two meshes are used for the computations presented hereafter. The first one of $2.74 \cdot 10^{5}$ vertices is a radial mesh with a first layer of vertices of thickness $\delta / 5$, and with a uniform meshing along the azimuthal and spanwise directions. The second one involves $1.2 \cdot 10^{6}$ vertices. It is also a radial mesh, but with a mesh clustering in the azimuthal direction which results in a mesh 4 times finer near the separation and the wake. The WALE SGS model is used for all the computations.

Let us analyze the case at $R e=10^{6}$. The main outputs are summed up in Table 2. Among the few measurements, we have reported the ones of Shih et al. [30], Schewe [29], Guven [13], [35], more recently Gölling [10], and the synthetic book of Zdravkovich [40]. We have found only three computations with LES models, by Kim and Mohan [17] and Catalano et al. [5], Ono and Tamura [24], and a RANS result in [5]. Rather surprisingly, our RANS calculation give results which differ importantly with the RANS results produced in [5] with a similar model. Our RANS prediction of drag is rather good, but the Strouhal predicted is a little high with respect to experiments, and the $C_{l}^{\prime}$ is 2-3 times too low. The LES simulations of the above references are carried out on grids having $2.3 \cdot 10^{6}[5], 4.5 \cdot 10^{6}$ nodes [24], and $6.8 \cdot 10^{6}$ nodes [17]. We check that LES computations need a rather high number of nodes, since with less than $4 \mathrm{M}$ nodes, mean drag is over estimated. The fine mesh calculations give a rather coherent prediction of the $C_{l}^{\prime}$, at $0.12-0.13$. With our very coarse mesh the VMS/LES calculation gives values of drag and $C_{l}^{\prime}$ too high. The hybrid RANS/VMS-LES simulations give rather accurate predictions in terms of mean drag, base pressure and Strouhal number already on the coarser grid, while the VMS/LES one on the same grid gives a significant overestimation of the mean drag coefficient. The effect of grid refinement on the predictions of the bulk parameters obtained in the hybrid RANS/VMS-LES simulations are moderate, except for the time fluctuations of lift. The appraisal of the results for this parameter is not evident, we adopt the range of $[0.1-0.15]$ indicated by Zdravkovich . A reasonably high level of fluctuation appears in instantaneous views of velocity and vorticity (Figures 6 and 7).

For comparison purpose with available $C_{p}$ measurements [27, 38], we have recomputed the same flow through the hybrid RANS/VMS-LES approach at Reynolds numbers $6.7 \cdot 10^{5}$ and $1.25 \cdot 10^{6}$, see Figure 5 . The overall agreement 
is good. Note again how grid refinement has a limited effect on the mean pressure output. For the case at $R e=6.7 \cdot 10^{5}$ in Figure 5 a, there is a slight under-estimation of the base pressure. Moreover, in the experimental curve there is a kink at $\theta=110^{\circ}$, which indicates the presence of a small secondary recirculation bubble. This bubble is not captured in our calculations. It is not either captured in [5], but a separation is found in [24]. At $R e=10^{6}$ the base pressure is very well predicted, while it seems that the separation occurs upstream than in the experiments. However, the prediction of the separation angle obtained at $R e=10^{6}, \theta=127^{\circ}$, is not so different from the rather recent measurement $\theta=130^{\circ}$ obtained by Goelling [10].

Table 2: Bulk flow parameters prediction for simulations at Rey $=10^{6}: \bar{C}_{d}$ is the mean drag, $C_{l}^{\prime}$ is the root mean square (r.m.s) of the lift coefficient, $\theta$ is the separation angle, $S t$ the vortex shedding frequency made nondimensional by the cylinder diameter and the freestream velocity.

\begin{tabular}{|c|c|c|c|c|c|c|}
\hline Present simulations & $\begin{array}{c}\text { Mesh } \\
\text { size }\end{array}$ & $\overline{C_{d}}$ & $C_{l}^{\prime}$ & $C p_{\text {base }}$ & St & $\theta$ \\
\hline $\begin{array}{l}\text { URANS } \\
\text { LES-VMS } \\
\text { Hybrid RANS } \\
\end{array}$ & $\begin{array}{l}1.2 \mathrm{M} \\
1.2 \mathrm{M} \\
1.2 \mathrm{M} \\
\end{array}$ & $\begin{array}{l}0.24 \\
0.36 \\
0.24 \\
\end{array}$ & $\begin{array}{l}0.06 \\
0.22 \\
0.17 \\
\end{array}$ & $\begin{array}{l}0.25 \\
0.22 \\
0.28 \\
\end{array}$ & $\begin{array}{c}0.46 \\
0.38 / 0.17 \\
\end{array}$ & $\begin{array}{l}129 \\
118 \\
\end{array}$ \\
\hline \multicolumn{7}{|l|}{ Simulations } \\
\hline $\begin{array}{l}\text { RANS of Catalano et al. [5] } \\
\text { LES of Catalano et al. [5] } \\
\text { LES of Ono and Tamura [24] } \\
\text { LES of Kim and Mohan [17] }\end{array}$ & $\begin{array}{l}2.3 \mathrm{M} \\
2.3 \mathrm{M} \\
4.5 \mathrm{M} \\
6.8 \mathrm{M}\end{array}$ & $\begin{array}{l}0.39 \\
0.31 \\
0.27 \\
0.27\end{array}$ & $\begin{array}{l}0.13 \\
0.12\end{array}$ & $\begin{array}{l}0.33 \\
0.32 \\
0.28\end{array}$ & $\begin{array}{c}0.35 \\
0.4 \\
-\end{array}$ & 108 \\
\hline \multicolumn{7}{|l|}{ Experiments } \\
\hline $\begin{array}{l}\text { Shih et al. [30] } \\
\text { Schewe [29] } \\
\text { Szechenyi [35] } \\
\text { Guven et al. [13] } \\
\text { Goelling [10] } \\
\text { Zdravkovich [40] }\end{array}$ & & $\begin{array}{l}0.24 \\
0.22 \\
0.25 \\
0.22 \\
\\
0.2-0.4\end{array}$ & $.1-.15$ & $\begin{array}{l}0.33 \\
0.32\end{array}$ & $\begin{array}{c}0.44 \\
0.35 \\
0.35 / 0.10 \\
0.50 / 0.18\end{array}$ & 130 \\
\hline
\end{tabular}




\section{Concluding remarks}

A strategy for blending RANS and VMS-LES has been investigated in the simulation of the flow around a circular cylinder. A blending function is introduced, in order to automatically switch from RANS to LES and vice versa. For complex geometries and complex grid topologies, as e.g. for unstructured grids, this automatic switch may be advantageous with respect to methods in which RANS and LES regions are a priori specified. The blending function is based on the values of a blending parameter. Different definitions of this parameter are proposed and investigated herein; all of them are aimed to identify whether the used grid is adequate to LES, i.e. if it is adequate to resolve additional fluctuations with respect to RANS, while the distance from the wall is not explicitly used. This again is interesting for complex geometries and complex grid topologies, as for unstructured grids (used herein), for which the computation of the distance from the wall yields practical difficulties. Another positive feature is that the investigated strategy does not require any specific RANS or LES closure. In particular, in the present paper, the VMS approach has been integrated in the hybridization strategy for the closure of the LES part, with different eddy-viscosity subgrid scale models.

This hybrid method has been applied to the simulation of the flow around a circular cylinder at Reynolds number $1.4 \cdot 10^{5}$, characterized by turbulent boundary-layer separation due to ad-hoc inflow conditions. The sensitivity to different parameters, viz. the grid resolution, the SGS model for the LES closure and the definition of the blending parameter, has been investigated. The results are practically insensitive to the definition of the blending parameter. For all the considered definitions, the model works in RANS mode in the boundary layer and in the shear layers detaching from the cylinder while LES mode is used in the wake, passing through a layer in which the model works in hybrid way. This gives an a posteriori support to our choice of not explicitly enforcing the distance from the wall in the definition of the blending parameter. The predictions of the main flow parameters are in good agreement with the results of DES simulations in the literature. For much higher Reynolds numbers, from $6.7 \cdot 10^{5}$ to $1.25 \cdot 10^{6}$, bulk quantities and the mean $\mathrm{Cp}$ distribution over the cylinder can be reasonably predicted even with the use of a very coarse mesh in the case of the RANS/VMS-LES hybridization. Grid refinement has only a moderate impact on the hybrid RANS/VMS-LES predictions. These results are encouraging in the perspec- 
tive of the application of the same methodology to the simulation of complex engineering problems, such as flows over tube arrays or components of offshore platforms.

\section{Acknowledgements}

This work has been supported by French National Research Agency (ANR) through COSINUS program (project ECINADS n ${ }^{\circ}$ ANR-09-COSI-003). HPC resources from GENCI-[CINES] (Grant 2010-x2010026386 and 2010c2009025067) and from CINECA (Bologna) and CASPUR are also gratefully acknowledged.

\section{References}

[1] E. Achenbach. Distribution of local pressure and skin friction around a circular cylinder in cross-flow up to $\mathrm{Re}=5 \times 10^{6}$. J. Fluid Mech., 34(4):625-639, 1968.

[2] P. Batten, U. Goldberg, and S. Chakravarthy. Interfacing statistical turbulence closures with large-eddy simulation. AIAA Journal, 42(3):485492, 2004.

[3] R.A. Baurle, C.-J. Tam, J.R. Edwards, and H.A. Hassan. Hybrid simulation approach for cavity flows: Blending, algorithm, and boundary treatment issues. AIAA J., 41(8):1463-1480, 2003.

[4] S. Camarri, M. V. Salvetti, B. Koobus, and A. Dervieux. A low diffusion MUSCL scheme for LES on unstructured grids. Comp. Fluids, 33:11011129, 2004.

[5] P. Catalano, Meng Wang, G. Iaccarino, and P. Moin. Numerical simulation of the flow around a circular cylinder at high Reynolds numbers. Int. J. Heat Fluid Flow, 24:463-469, 2003.

[6] M. Jaeger G. Dhatt. An extended $k-\varepsilon$ finite element model. Int. J. for Numerical Methods in Fluids, 14:1325-1345, 1992.

[7] A. Fadai-Ghotbi, Ch. Friess, R. Manceau, T. Gatski, and J. Borée. Temporal filtering: a consistent formalism for seamless hybrid ransles modeling in inhomogeneous turbulence. Int. J. Heat Fluid Flow, 31(3), 2010. 
[8] C.-C. Fan, X. Xiao, J.R. Edwards, and H.A. Hassan. Hybrid largeeddy/Reynolds-averaged navier-stokes simulations of shock-separated flows. J. Spacecraft Rockets, 41(6):897-906, 2004.

[9] J. Froehlich and D. von Terzi. Hybrid les/rans methods for the simulation of turbulent flows. Prog. Aerosp. Sci., 44(5):349-377, 2008.

[10] B. Goelling. Experimental investigations of separating boundary-layer flow from circular cylinder at reynolds numbers from $10^{5}$ up to $10^{7}$; three-dimensional vortex flow of a circular cylinder. In G.E.A. Meier and K.R. Sreenivasan, editors, Proceedings of IUTAM Symposium on One Hundred Years of Bloundary Layer Research, pages 455-462, The Netherlands, 2006. Springer.

[11] U. Goldberg, O. Peroomian, and S. Chakravarthy. A wall-distance-free $k-\varepsilon$ model with enhanced near-wall treatment. J. Fluids Eng., 120:457462, 1998.

[12] H. Guillard and C. Viozat. On the behaviour of upwind schemes in the low Mach number limit. Comp. Fluids, 28:63-86, 1999.

[13] O. Guven, C. Farell, and V.C. Patel. Surface-roughness effects on the mean flow past circular cylinders. J. Fluid Mech., 98(4):673-701, 1980.

[14] J. Hinze. Turbulence. MacGraw-Hill, New York, 1959.

[15] W.D. James, S.W. Paris, and G.V. Malcolm. Study of viscous cross flow effects on circular cylinders at high Reynolds numbers. AIAA J., 18:1066-1072, 1980.

[16] S. Kawai and K. Fujii. Analysis and prediction of thin-airfoil stall phenomena with hybrid turbulence methodology. AIAA J., 43(5):953-961, 2005.

[17] S.-E. Kim and L.S. Mohan. Prediction of unsteady loading on a circular cylinder in high reynolds number flows using large eddy simulation. Proceedings of OMAE 2005: 24th International Conference on Offshore Mechanics and Artic Engineering, june 12-16, Halkidiki, Greece, OMAE 2005-67044, 2005. 
[18] B. Koobus and C. Farhat. A variational multiscale method for the large eddy simulation of compressible turbulent flows on unstructured meshes-application to vortex shedding. Comput. Methods Appl. Mech. Eng., 193:1367-1383, 2004.

[19] E. Labourasse and P. Sagaut. Reconstruction of turbulent fluctuations using a hybrid RANS/LES approach. J. Comp. Phys., 182:301-336, 2002 .

[20] S.-C. Lo, K.A. Hofmann, and J.-F. Dietiker. Numerical investigation of high Reynolds number flows over square and circular cylinder. $J$. Thermophys. Heat Trans., 19:72-80, 2005.

[21] T. Marcel, M. Braza, Y Hoarau, G. Haran, F. Baj, J.P. Magnaud, and E. Longatte. Simulation of fluid-structure interaction in a tube array under cross flow at high reynolds numver. ERCOFTAC Bulletin, 86:10:5459, 2011.

[22] R. Mittal and S. Balachandar. Effect of three-dimensionality on the lift and drag of nominally two-dimensional cylinders. Phys. Fluids, 7(8):1841-1865, 1995.

[23] F. Nicoud and F. Ducros. Subgrid-scale stress modelling based on the square of the velocity gradient tensor. Flow Turb. Combustion, 62(3):183-200, 1999.

[24] Y. Ono and T. Tamura. Les of flows around a circular cylinder in the critical reynolds number region. In Proceedings of BBAA VI International Colloquium on: Bluff Bodies Aerodynamics and Applications, Milano, Italy, July 20-24 2008.

[25] H. Ouvrard, B. Koobus, A. Dervieux, and M.V. Salvetti. Classical and variational multiscale large-eddy simulations of the flow around a circular cylinder on unstructured grids. Computer and Fluids, 39:1083-1094, 2010.

[26] P.L. Roe. Approximate Riemann solvers, parameters, vectors and difference schemes. J. Comp. Phys., 43:357-372, 1981.

[27] A. Roshko. Experiments on the flow past a circular cylinder at very high reynolds number. J. Fluid. Mech., 10:345-356, 1961. 
[28] P. Sagaut, S. Deck, and M. Terracol. Multiscale and multiresolution approaches in turbulence. Imperial College Press, London, 2006.

[29] J.W. Schewe. On the forces acting on a circular cylinder in cross flow from subcritical up to transcritical Reynolds numbers. J. Fluid Mech., 133:265-285, 1983.

[30] W.C.L. Shih, C. Wang, D. Coles, and A. Roshko. Experiments on flow past rough circular cylinders at large reynolds numbers. J. Wind Eng. Indust. Aerodyn., 49:351-368, 1993.

[31] S. Sirnivas, S. Wornom, A. Dervieux, B. Koobus, and O. Allain. A study of LES models for the simulation of a turbulent flow around a truss spar geometry. In Proceedings of OMAE'06, 25 ${ }^{\text {rd }}$ International Conference on Offshore and Arctic Engineering, number OMAE2006-92355, Hamburg, Germany, 2006.

[32] J. Smagorinsky. General circulation experiments with the primitive equations. Monthly Weather Review, 91(3):99-164, 1963.

[33] P.R. Spalart, W.H. Jou, M. Strelets, and S. Allmaras. Advances in DNS/LES, chapter Comments on the feasibility of LES for wings and on a hybrid RANS/LES approach. Columbus (OH), 1997.

[34] B.M. Sumer and J. Fredsoe. Hydrodynamics around cylindrical structures. World Scientific, 2006.

[35] E. Szechenyi. Supercritical reynolds number simulation for twodimensional flow over circular cylinders. J. Fluid Mech., 70:529-542, 1975 .

[36] A. Travin, M. Shur, M. Strelets, and P. Spalart. Detached-eddy simulations past a circular cylinder. Flow Turb. Combustion, 63:293-313, 1999.

[37] A.W. Vreman. An eddy-viscosity subgrid-scale model for turbulent shear flow: algebraic theory and application. Phys. Fluids, 16:3670-3681, 2004 .

[38] K. A. Warschauer and J. A. Leene. Experiments on mean and fluctuating pressures of circular cylinders at cross flow at very high reynolds 


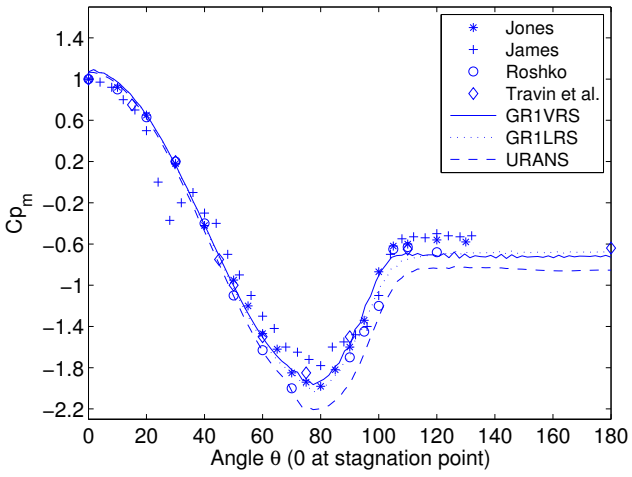

(a)

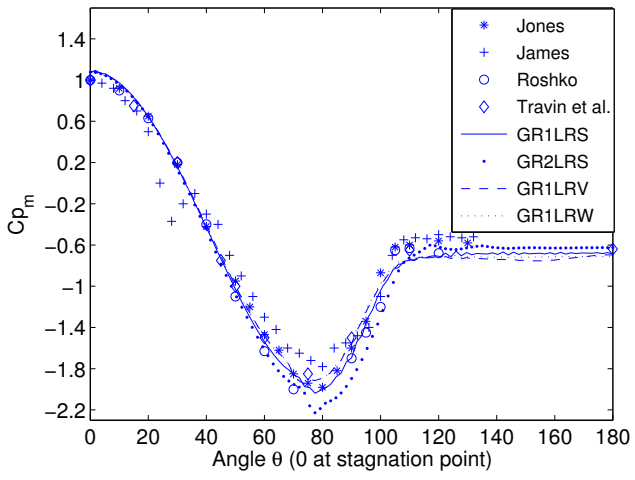

(b)

Figure 1: Flow past a cylinder at $1.4 \cdot 10^{5}$ : Distribution over the cylinder surface of the mean pressure coefficient obtained in the different simulations compared to experimental data and to DES results. See Table 1 for detailed legend.

numbers. Proc. Int. Conf. on Wind Effects on Buildings and Structures, Saikon, Tokyo, 305-315 (see also Zdravkovich 1997), 1971.

[39] S. Wornom, H. Ouvrard, M.-V. Salvetti, B. Koobus, and A. Dervieux. Variational multiscale large-eddy simulations of the flow past a circular cylinder : Reynolds number effects. Comp. Fluids, pages 44-50, 2011.

[40] M.M. Zdravkovich. Flow around circular cylinders. Vol 1: Fundamentals. Oxford Science Publications. Oxford University Press, 1997. 


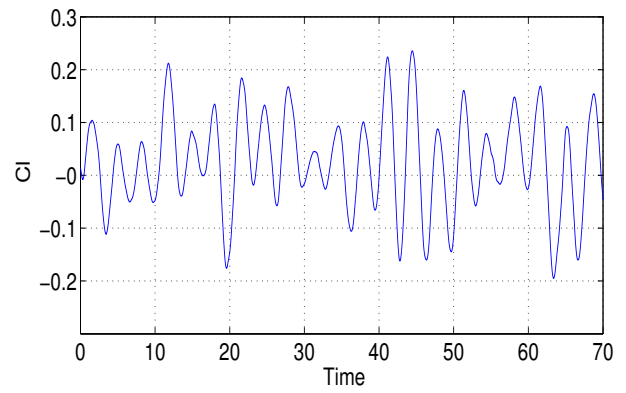

(a)

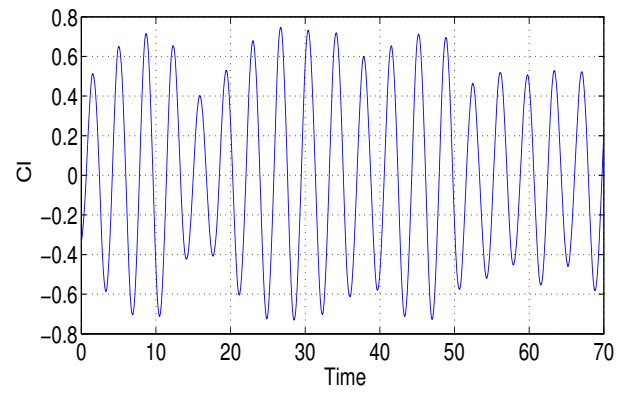

(b)

Figure 2: Flow past a cylinder at $1.4 \cdot 10^{5}$ : Time variation of the lift coefficient. (a) GR1-LRS (coarse grid, blending on length ratio, VMS with Smagorinsky SGS); (b) GR1URANS.

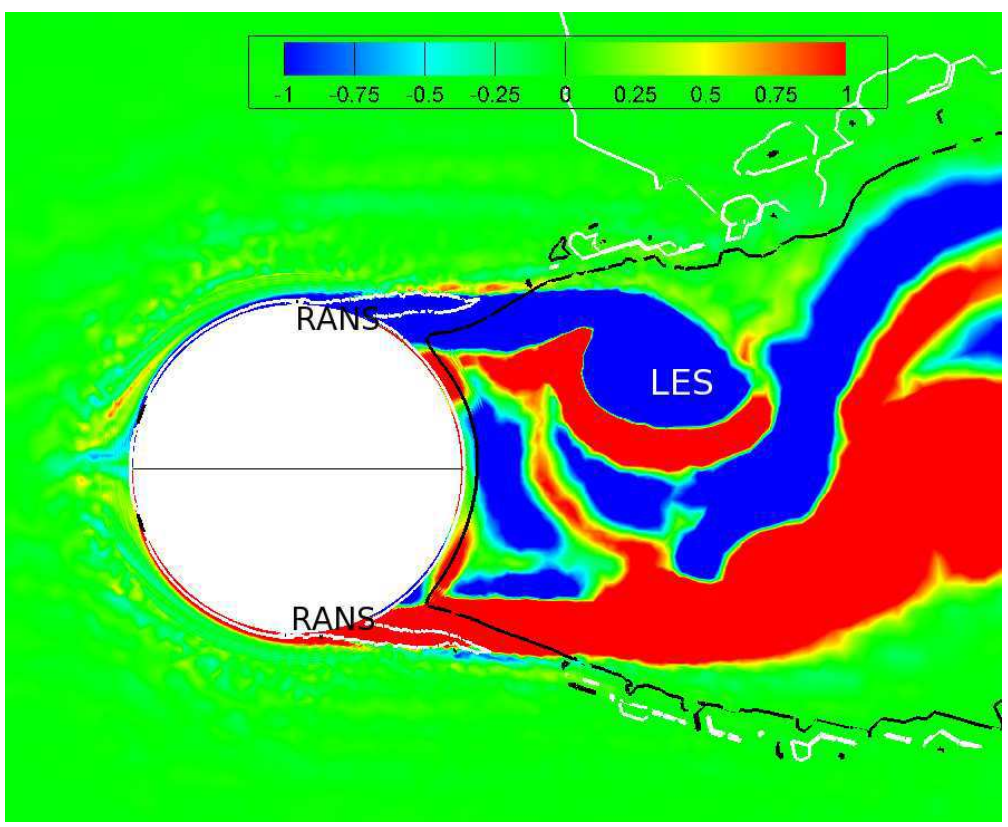

Figure 3: Flow past a cylinder at $1.4 \cdot 10^{5}$ :Instantaneous isocontours of spanwise vorticity (simulation GR1-LRS). The lines are the isolines of the blending function: $\theta=0.1$ (black) and $\theta=0.9$ (white). 

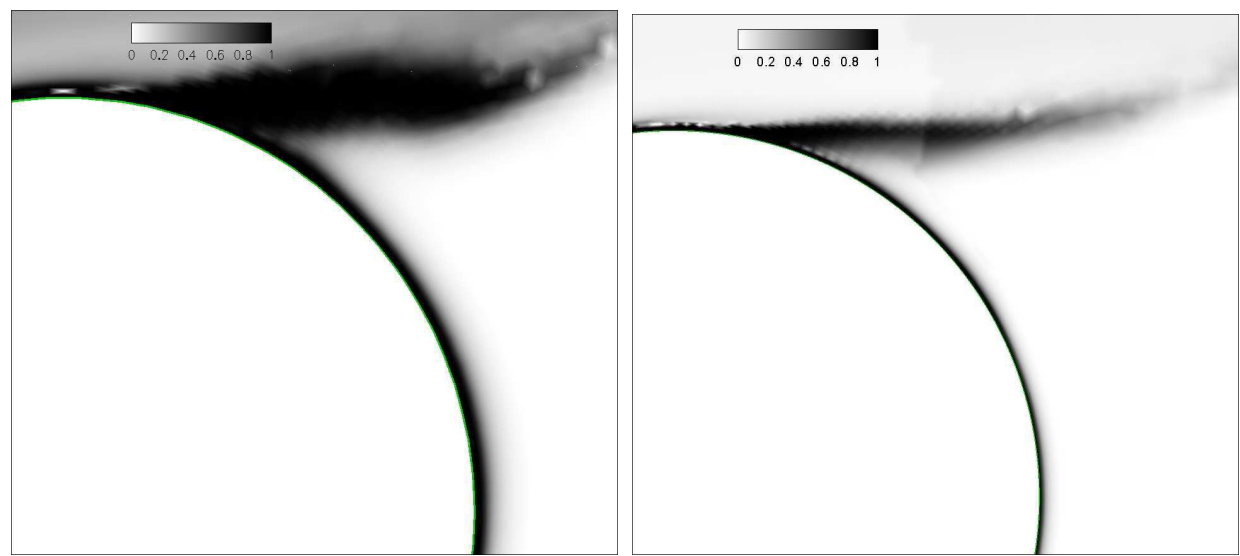

Figure 4: Flow past a cylinder at $1.4 \cdot 10^{5}$ : Instantaneous isocontours of the blending function $\theta$ based on characteristic length ratio. The isocontours range from 0 (white), corresponding to a full LES correction, to 1 (black), corresponding to pure RANS. Simulations GR1-LRS (left) and GR2-LRS (right).

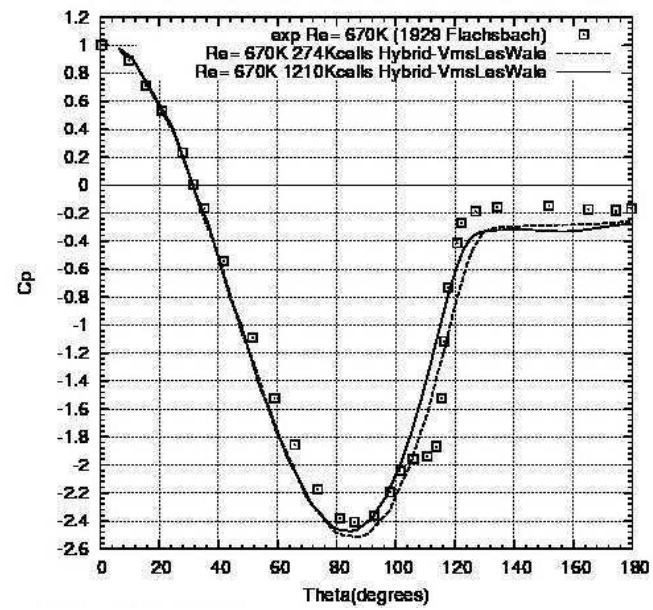

(a)

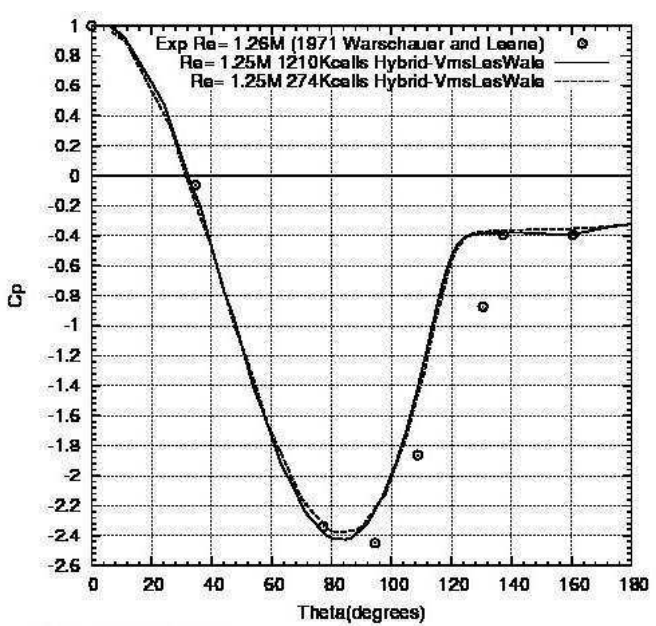

(b)

Figure 5: Mean $C_{p}$ as a function of polar angle. Comparison between experimental data and numerical results obtained on two meshes, of resp. $2.74 \cdot 10^{5}$ cells and $1.21 \cdot 10^{6}$ cells. (a) Reynolds $6.7 \cdot 10^{5}$, present results compared with measurements of Flachsbart [27]. (b) Reynolds $1.25 \cdot 10^{6}$ comparison with measurements of Warschauer and Leene [38]. 


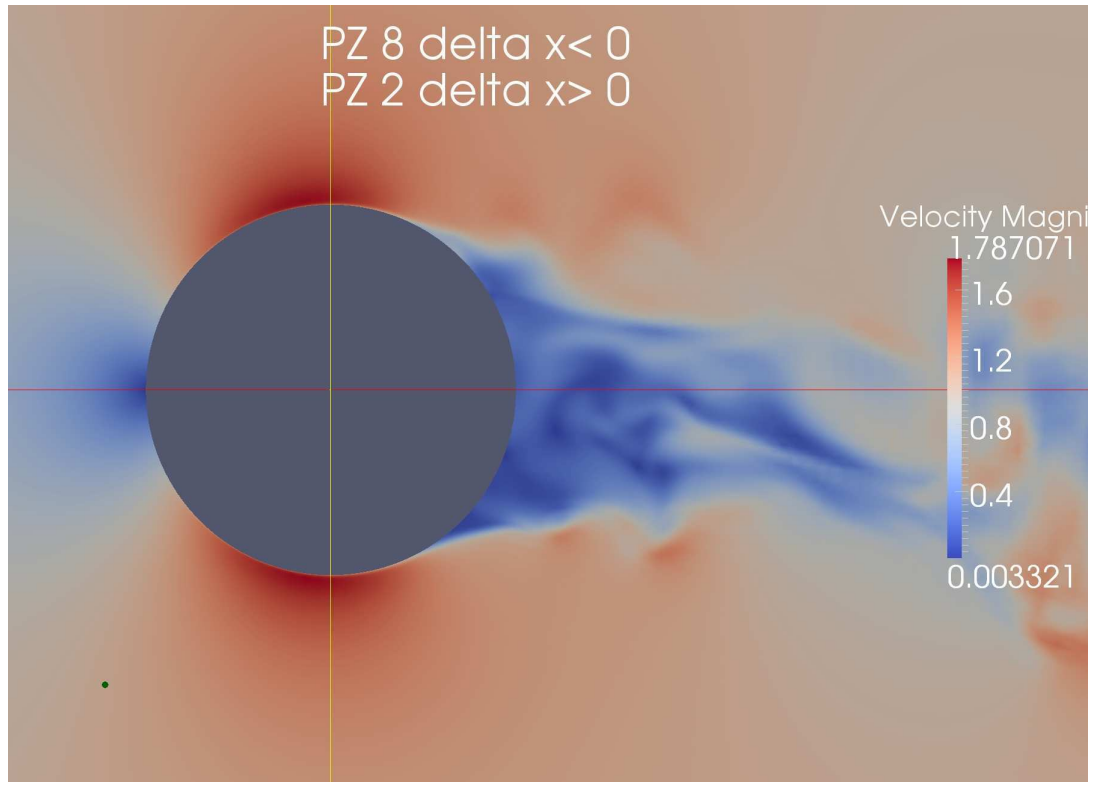

Figure 6: Flow around a cylinder at Reynolds number 1M : instantaneous views of velocity.

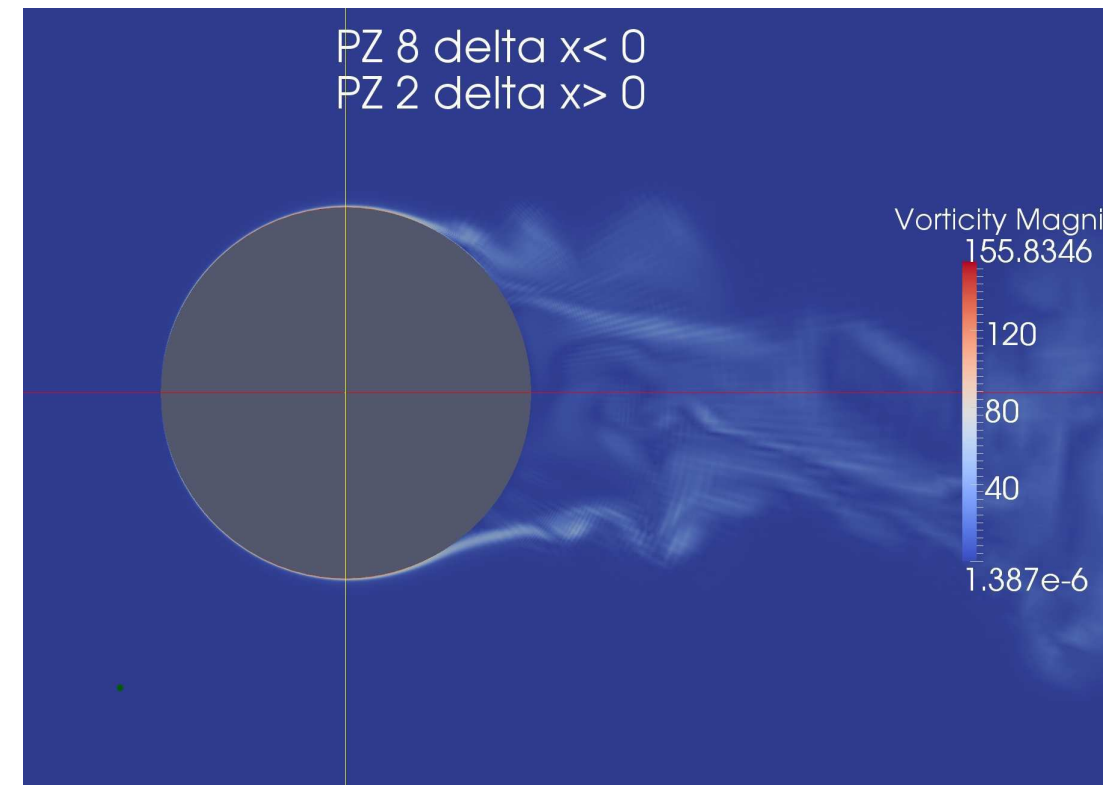

Figure 7: Flow around a cylinder at Reynolds number $1 \mathrm{M}$ : instantaneous views of vorticity. 